LLNL/Rochester 2007 TRIUMF activity

C.Y. Wu, J.A. Becker, A.M. Hurst, M.A. Stoyer, D. Cline, A.B. Hayes

August 30, 2007 
This document was prepared as an account of work sponsored by an agency of the United States Government. Neither the United States Government nor the University of California nor any of their employees, makes any warranty, express or implied, or assumes any legal liability or responsibility for the accuracy, completeness, or usefulness of any information, apparatus, product, or process disclosed, or represents that its use would not infringe privately owned rights. Reference herein to any specific commercial product, process, or service by trade name, trademark, manufacturer, or otherwise, does not necessarily constitute or imply its endorsement, recommendation, or favoring by the United States Government or the University of California. The views and opinions of authors expressed herein do not necessarily state or reflect those of the United States Government or the University of California, and shall not be used for advertising or product endorsement purposes.

This work was performed under the auspices of the U.S. Department of Energy by University of California, Lawrence Livermore National Laboratory under Contract W-7405-Eng-48. 


\title{
I. Bambino: a segmented silicon detector system for TIGRESS
}

\author{
C.Y. Wu, J.A. Becker, A.M. Hurst, and M.A. Stoyer \\ Lawrence Livermore National Laboratory, Livermore, CA 94550 \\ D. Cline and A.B. Hayes \\ Department of Physics, University of Rochester, Rochester, NY 14627
}

\begin{abstract}
Bambino is a first generation auxiliary detector for the TIGRESS array and consists of a pair of segmented annular silicon detectors, fabricated by MicronSemiconductor Inc. Bambino provides measures of both the energy and position for outgoing charged particles and main triggers for valid events. The annular Bambino detectors are placed $3.0 \mathrm{~cm}$ from the target both upstream and downstream. Each detector has 24 rings in $\theta$ covering angles between $20.1^{\circ}$ and $49.4^{\circ}$ and between $130.6^{\circ}$ to $159.9^{\circ}$, with 16 sectors in $\phi$ for $2 \pi$ coverage. Two sets of preamplifiers, manufactured by SwanResearch, with the sensitivity of either 5 or $50 \mathrm{mV} / \mathrm{MeV}$ are available for experiments. A special scattering chamber to accommodate this detector array was designed and fabricated in FY06 by U. of Rochester. Bambino functioned very well for the first successful TIGRESS experiment on the Coulomb excitation of radioactive ${ }^{20} \mathrm{Na}$ and ${ }^{21} \mathrm{Na}$ beams in Jul/Aug 2006.
\end{abstract}

Bambino underwent a major upgrade in FY07 at a cost of about $\$ 80 \mathrm{k}$ to increase the position resolution. This is achieved by doubling the number of sector to 32 and will improve the in-flight reaction product $\gamma$-ray spectroscopy resolution to about $1 \%$ or better for the TIGRESS array. This new segmented silicon detector, called CD-S3, was designed and fabricated by MicronSemiconductor Inc. CD-S3 was installed and used for the scheduled experiment E1075 in Jul/Aug 2007 (Coulomb excitation of radioactive ${ }^{29} \mathrm{Na}$ beam). In addition, assemble of a $\Delta \mathrm{E}-\mathrm{E}$ detector array for the light charged-particle identification is taking place for future experiments by acquiring detectors with thickness of both $\sim 140$ and $\sim 1,000 \mu \mathrm{m}$ and associated preamplifiers. 


\section{E1075: Shell gap narrowing in ${ }^{29} \mathrm{Na}$}

The disappearance of the $N=20$ magic number ( $s d$ to $p f$ shells) in neutron-rich nuclei with mass near 30, the so-called "island of inversion", is a well-known phenomenon. This shell gap collapse is a direct consequence of the modification of residual interactions in these neutron-rich nuclei. However, a quantitative description of the transition from a $s d$ shell-dominated to a $p f$-shell-dominated configuration for ground states of these nuclei is missing. Neutron-rich $\mathrm{Na}$ isotopes provide a unique opportunity to study this transition quantitatively. For instance, the ground state of ${ }^{28} \mathrm{Na}$ has a $s d$-shell-dominated configuration while the ground state of ${ }^{30} \mathrm{Na}$ has a $p f$-shell-dominated configuration. A significant mixing between $s d$ and $p f$ shells for the ground-state configuration of ${ }^{29} \mathrm{Na}$ is implied from the recent $\beta$-decay study of ${ }^{29} \mathrm{Ne}$. The goal of E1075 is to study the groundstate configuration of ${ }^{29} \mathrm{Na}$ through the inferred quadrupole deformation from the measured E2 transition rate of the first excited state using the Coulomb-excitation technique. Studying the electromagnetic properties of given states is a direct measure of their wavefunctions. In addition, modern shell model codes are beginning to incorporate the $s d-p f$ configuration space in a realistic way, and comparison of the measured electromagnetic transition moments with the predictions provides an excellent discrimination of the nuclear theory embodied in these codes.

The experiment was carried out at ISAC II in Jul/Aug 2007 using TIGRESS/Bambino by bombarding $70 \mathrm{MeV}{ }^{29} \mathrm{Na}$ on a $2.94 \mathrm{mg} / \mathrm{cm}^{2}{ }^{110} \mathrm{Pd}$ target. The deexcitation $\gamma$ rays were detected by six segmented clover detectors of the TIGRESS array in coincidence with the detection of scattered or recoiling particles by the Bambino CD-S3 detector, mounted downstream. The radioactive beam, composed of both ${ }^{29} \mathrm{Al}$ and ${ }^{29} \mathrm{Na}$, was delivered with an intensity that varied between 400 and $800 \mathrm{p} / \mathrm{s}$ during the experiment. Beam composition is an issue and was monitored continuously at the beam dump by measuring characteristic $\gamma$ rays following the $\beta$ decay. However during the course of this experiment, we discovered that the separation of ${ }^{29} \mathrm{Na}$ from ${ }^{29} \mathrm{Al}$ can be accomplished by their energy loss difference in the ${ }^{110} \mathrm{Pd}$ target, which was observed in the energy spectrum obtained by the Bambino CD-S3 detector and shown in Fig. 1. This is the first time that isobar separation has been achieved by a thin-foil technique. The sensitivity and selectivity of TIGRESS/Bambino can be demonstrated in Fig. 2, where the coincident $\gamma$ rays from the deexcitation of both ${ }^{29} \mathrm{Na}$ and ${ }^{110} \mathrm{Pd}$ were observed despite the extremely low beam intensity. Background $\gamma$-ray activity detected by TIGRESS amounts $\sim 1.2 \mathrm{kHz}$ compared to $\sim 0.001 \mathrm{~Hz}$ coincident rate induced by the available beam on target. The full data analysis of this experiment will be carried out at LLNL. 


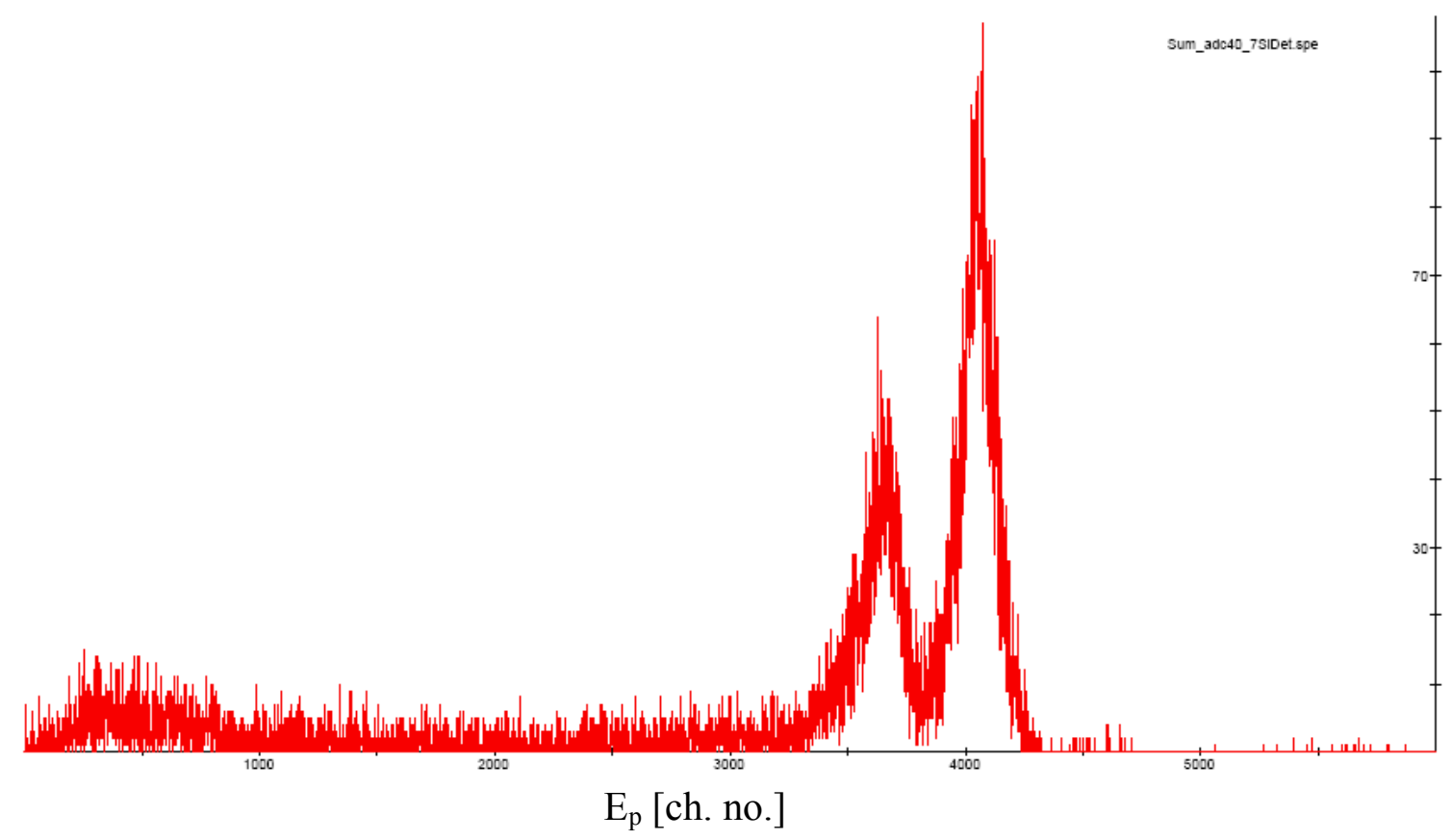

Fig. 1 The energy spectrum of scattered particles from a $2.94 \mathrm{mg} / \mathrm{cm}^{2}{ }^{110} \mathrm{Pd}$ target at $\sim 21^{\circ}$. The peak at left is ${ }^{29} \mathrm{Al}$ and the peak at right is ${ }^{29} \mathrm{Na}$. This separation results from the difference of their energy loss in the ${ }^{110} \mathrm{Pd}$ target.

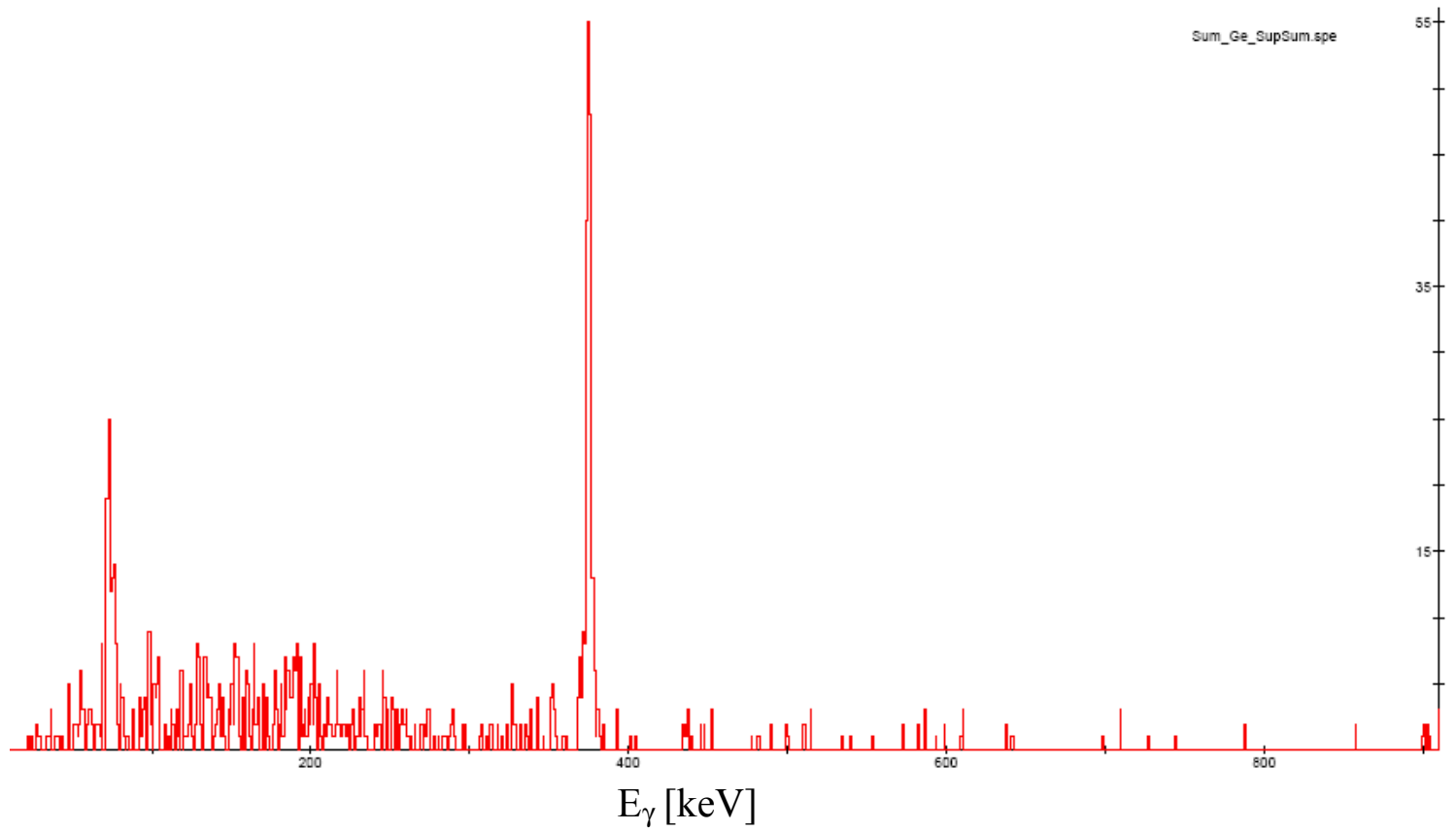

Fig. 2 The $\gamma$-ray spectrum obtained by the TIGRESS array in coincidence with the detection of scattered particles by Bambino. The $5 / 2^{+} \rightarrow 3 / 2^{+}$transition $(72 \mathrm{keV})$ in ${ }^{29} \mathrm{Na}$ is shown at left. The $2^{+} \rightarrow 0^{+}$transition $(374 \mathrm{KeV})$ in ${ }^{110} \mathrm{Pd}$ is shown at right. 


\section{SuperCHICO: a pixelated parallel-plate avalanche counter}

The next generation of $\gamma$-ray energy tracking arrays with segmented Ge crystals, such as TIGRESS and GRETINA, have begun operation in an early implementation mode. Their achieved geometrical resolution creates a real demand for a new generation of auxiliary charged particle detectors with a matching position resolution to fully realize the potential of these arrays. We propose development of a new generation of parallel-plate positionsensitive avalanche counter for the heavy-ion detection with a design goal of $\approx 2 \mathrm{~mm}$ position resolution. This position resolution is required so that the experimental $\gamma$-ray resolution for in-flight reaction products smeared by Doppler shift can be recovered to match the resolution of $\gamma$ rays emitted by nuclei at rest. This proposed detector system, named SuperCHICO, has been identified as one of the essential auxiliary detectors for the GRETA array stated in the "GRETA: The Future of Gamma-Ray Spectroscopy" 2007 white paper. (http://grfs1.lbl.gov/DocDB/0001/000139/001/GRETA_WP Jan06 4.pdf)

SuperCHICO follows the design of the highly successful CHICO gas avalanche counter with two new developments: 1. the pixelated cathode board for two-dimensional position read-out, 2. the digitizer to process the signal instead of the conventional analog modules. To have an early implementation of SuperCHICO in conjunction with TIGRESS, we have submitted a budget plan of $\$ 1.34 \mathrm{M}$ to US DOE for the construction of SuperCHICO beginning in FY10. Note that this budget request is for the planning purpose and does not constitute an approval of the construction of SuperCHICO. A R\&D proposal for SuperCHICO together with other auxiliary detectors associated with GRETA has been submitted in May 2007 to US DOE. This R\&D is an important step toward the final design of SuperCHICO by providing proof-of-principle of the new pixelated board. This work will be carried out jointly by U. of Rochester and LLNL.

This work was performed under the auspices of the U.S. Department of Energy by the University of California, Lawrence Livermore National Laboratory under contracts no. W-7405-ENG-48. The work performed at University of Rochester was supported by NSF and AFOSR.

This document was prepared as an account of work sponsored by an agency of the United States Government. Neither the United States Government nor the University of California nor any of their employees, makes any warranty, express or implied, or assumes any legal liability or responsibility for the accuracy, completeness, or usefulness of any information, apparatus, product, or process disclosed, or represents that its use would not infringe privately owned rights. Reference herein to any specific commercial product, process, or service by trade name, trademark, manufacturer, or otherwise, does not necessarily constitute or imply its endorsement, recommendation, or favoring by the United States Government or the University of California. The views and opinions of authors expressed herein do not necessarily state or reflect those of the United States Government or the University of California, and shall not be used for advertising or product endorsement purposes. 